\title{
IMPLEMENTAÇÃO DE MEDIDAS PARA O ALÍVIO DA DOR EM NEONATOS PELA EQUIPE DE ENFERMAGEM
}

\author{
Nursing team implementation of measures for pain relief in neonates \\ Implementación de medidas para el alivio del dolor en neonatos por el equipo de \\ enfermería
}

Roberta Meneses Oliveira

Ana Paula Almeida Dias da Silva ${ }^{4}$
Ana Valeska Siebra e Silva²

Edna Maria Camelo Chaves $^{5}$
Lucilane Maria Sales da Silva ${ }^{3}$

Samara Cavalcante Bezerra ${ }^{6}$

\section{RESUMO}

Estudo transversal com abordagem quantitativa, que objetivou identificar a implementação de medidas para o alívio da dor em neonatos pelos profissionais de Enfermagem, bem como caracterizá-las em tipo, frequência e finalidade da aplicação. Desenvolvido entre agosto e outubro de 2007 em quatro hospitais de referência no atendimento neonatal em Fortaleza-Ceará. Um formulário foi aplicado em 180 profissionais. Os dados foram analisados com estatística descritiva simples e apresentados em tabelas. A maioria dos profissionais (98,8\%) afirmou implementar medidas para minimizar a dor do neonato, destacando-se: Chupeta de gaze com glicose (43,3\%); Acalento (23,3\%); Pacotinho (19,4\%). Quanto às justificativas, a maioria (85\%) relatou que executa tais medidas para acalmar/aliviar o sofrimento do bebê. Em conclusão, as entrevistadas demonstraram conhecer o efeito benéfico da glicose para o neonato e implementar estratégias que, aplicadas em conjunto antes dos procedimentos dolorosos, proporcionam alívio e tranquilidade para o bebê.

Palavras-chave: Dor. Analgesia. Recém-nascido. Enfermagem Neonatal. Cuidados Intensivos.

\section{Abstract}

It's a Cross-sectional study with a quantitative approach, aiming to identify nursing team implementation of pain relief measures in neonates and characterize them as the purpose, type and frequency. Data collection took place between August and October 2007 at four neonatal care reference hospitals in Fortaleza, Ceará. A form was answered by 180 professionals. Data were analyzed with descriptive statistics in tables. Most of professionals (98.8\%) reported to implement measures to minimize pain in the newborn, pinpointing: gauze with glucose $(43.3 \%)$; to tuck the baby (23.3\%); to involve the baby in this blanket (19.4\%). In a way of justification, the majority $(85 \%)$ reported implementing such measures to relieve the baby's suffering. It is concluded that the subjects interviewed knew about the beneficial effect of glucose on the neonate, and implement strategies that, applied before painful procedures, provide relief and comfort to the baby.

Keywords: Pain. Analgesia. Infant, Newborn. Neonatal Nursing. Intensive care.

\section{Resumen}

Estudio transversal con enfoque cuantitativo, que tuvo como objetivo identificar las medidas de ejecución para el alivio del dolor en recién nacidos por los profesionales de enfermería, bien cómo caracterizarlos por tipo, frecuencia y efectos de la aplicación. El estudio fue desarrollado en cuatro hospitales de referencia en la atención neonatal en Fortaleza-Ceará, entre agosto y octubre de 2007. Se aplicó una forma a 180 profesionales. Los datos fueron analizados con estadísticas descriptivas en tablas. La mayoría de los profesionales (98,8\%) afirmó aplicar medidas para minimizar el dolor del neonato, incluyendo: chupete de gasa con la glucosa $(43,3 \%)$; acunar el neonato $(23,3 \%)$, envolver el neonato $(19,4 \%)$. Como justificación, la mayoría (85\%) reporta realizar las medidas para aliviar el sufrimiento del bebé. En conclusión, las entrevistadas demostraron conocer el efecto beneficioso de la glucosa para el neonato y ejecutar estrategias que, utilizadas en conjunto antes de los procedimientos dolorosos, proporcionan alivio y confort para el recién nacido.

Palabras clave: Dolor. Analgesia. Recién nacido. Enfermería Neonatal. Cuidados intensivos.

\footnotetext{
'Enfermeira Especialista em Enfermagem Clínica. Aluna do Curso de Mestrado Acadêmico Cuidados Clínicos em Saúde (CMACCLIS) da Universidade Estadual do Ceará (UECE). Fortaleza - CE. Brasil. Email: menesesroberta@yahoo.com.br.²Enfermeira. Doutoranda em Saúde Pública pela USP, São Paulo - Brasil. Docente do Departamento de Enfermagem da UECE. Orientadora da pesquisa. Fortaleza - CE. Brasil. Email: anavaleska@usp.br. ${ }^{3}$ Enfermeira. Doutora em Enfermagem. Docente do CMACCLIS. Fortaleza - CE. Brasil. Email: lucilanemaria@yahoo.com.brEnfermeira. Mestranda em Cuidados Clínicos em Saúde (CMACCLIS). Docente do Curso de Enfermagem da Universidade de Fortaleza - UNIFOR. Fortaleza - CE Brasil. Email: anapauladias@unifor.br ${ }^{5}$ Enfermeira. Mestre em Cuidados Clínicos em Saúde (CMACCLIS). Doutoranda em Farmacologia pela Universidade Federal do Ceará - UFC. Fortaleza - CE Brasil. Email: ednacam3@ibestvip.com.br ${ }^{6}$ Enfermeira. Especialista em Enfermagem Clínica. Fortaleza - CE Brasil. Email: samarac84@yahoo.com.br
} 


\section{Medida para alívio da dor em Neonatos}

\section{INTRODUÇÃO}

$\mathrm{Na}$ unidade de terapia intensiva neonatal (UTIN), os neonatos são submetidos a estímulos dolorosos que resultam de inúmeros procedimentos invasivos, tanto diagnósticos quanto interventivos.

Essa elevada carga de estímulos implica efeitos negativos na organização comportamental e fisiológica do neonato hospitalizado, gerando, consequentemente, um estresse crônico, fazendo com que ele utilize suas reservas energéticas que seriam direcionadas para o seu crescimento, restabelecimento e desenvolvimento. ${ }^{1}$

Embora os profissionais de saúde concordem que os neonatos sejam capazes de responder a estímulos nociceptivos por meio de alterações orgânicas, fisiológicas e comportamentais e de sentir dor, ainda é notória a pouca utilização de analgesia nas UTINs. ${ }^{2}$ Acredita-se que um dos maiores fatores que podem contribuir para essa oligoanalgesia seja a falta de conhecimento sobre analgésicos e sobre o manejo da dor pelos profissionais responsáveis pelo cuidado destes pacientes.

De acordo com a Academia Americana de Pediatria (AAP) e a Sociedade Americana de Dor (SAD), ${ }^{3}$ a principal atitude dos profissionais de saúde perante a dor de um neonato submetido a procedimentos invasivos, sejam diagnósticos ou terapêuticos, deve ser baseada na antecipação, ou seja, deve levar em conta a intensidade da dor e a duração esperada, 0 contexto no qual ele se encontra inserido e o tipo de procedimento. Aspectos como estes apontam para a implementação de adequadas medidas de analgesia e de confor to do neonato, buscando eliminar ou, pelo menos, diminuir a sua sensação dolorosa.

A despeito das inúmeras publicações e consensos internacionais referentes à prevenção e ao tratamento da dor neonatal, ${ }^{3}$ este assunto é raramente abordado em cursos de graduação e pós-graduação de Enfermagem no Brasil, tornando-o distante da prática assistencial.

0 estudo desperta interesse diante de sua grande relevância, ao buscar a identificação das práticas dos enfermeiros no manejo dor do neonato, uma estratégia que pode contribuir para melhorar a avaliação e o tratamento da dor por parte da equipe, bem como para subsidiar a implantação/implementação de protocolos que sirvam de guias para o melhor desempenho na prática.

Assim, teve como objetivos identificar se os profissionais de Enfermagem atuantes nas UTINs de referência em FortalezaCE implementam medidas para o alívio da dor em neonatos; e caracterizar as medidas utilizadas, bem como a frequência com que são aplicadas e as justificativas atribuídas para esta aplicação na prática.

Consideramos que a presente pesquisa contribui para gerar reflexões acerca da atuação da Enfermagem no alívio da dor do recém-nascido e dos efeitos tardios desta.
Pode, também, ser um dispositivo para direcionar a implementação do cuidado da dor de forma efetiva, melhor qualificando essa atuação.

\section{REVISÃO LITERATURA}

Em neonatos, o sistema nociceptor já se encontra formado, porém as respostas destes aos estímulos dolorosos são inespecíficas e desorganizadas, visto que há mielinização incompleta do sistema nervoso e fraca modulação pelos mecanismos de controle endógeno da dor. Como resultado, a dor sentida pelo neonato é bem mais for te e aguda do que na criança maior ou no adulto, traduzindo-se em desconfor to físico, psíquico e sofrimento para os neonatos, incapazes de relatá-lo verbalmente. ${ }^{4}$

Além disso, a dor causa aos neonatos instabilidade hemodinâmica e aumenta os índices de morbimortalidade nessa faixa etária, com potenciais repercussões a cur to, médio e longo prazo.

Esta dor frequente e prolongada é prejudicial ao desenvolvimento do sistema nervoso e pode ameaçar a estabilidade fisiológica de crianças prematuras e doentes. Além disso, pode levar à ocorrência de alterações permanentes devido ao desenvolvimento da plasticidade do cérebro imaturo e ocasionar um desenvolvimento alterado do sistema de dor associado à diminuição do limiar de dor. ${ }^{5}$

Também podem ocorrer repercussões a longo prazo relacionadas a déficit neurológico, déficit de cognição e aprendizado, dificuldade de interação com a família e com a sociedade. ${ }^{2}$

A enfermagem, pela maior proximidade do paciente no desempenho de suas atividades assistenciais, tem se destacado na implementação de medidas de prevenção, redução ou eliminação do desconforto produzido por estímulos indesejáveis em unidades neonatais, principalmente quando se trata de procedimentos invasivos edolorosos.

Particularmente no contexto de cuidados de enfermagem, merecem destaque as medidas não farmacológicas para 0 alívio da dor. Consideradas técnicas não invasivas para o controle da dor, compreendem um conjunto de medidas de ordem educacional, física, emocional e comportamental, na sua maioria de baixo custo, fácil aplicação e com riscos de complicações pequenas. ${ }^{6}$

Pesquisadores e profissionais de neonatologia acreditam na efetividade de tais medidas, por promoverem estabilidade e boa organização do neonato, podendo ser úteis na conservação de energia para seu crescimento e desenvolvimento. ${ }^{7}$ Apesar disso, têm sido pouco utilizadas nas unidades neonatais, onde diária e continuamente são realizados inúmeros procedimentos extremamente dolorosos sem analgesia prévia. 


\section{METODOLOGIA}

Trata-se de uma pesquisa descritiva, transversal e de abordagem quantitativa, realizada em quatro hospitais públicos de referência no atendimento neonatal no Ceará. Estes são instituições de grande porte, que promovem assistência ao recémnascido gravemente enfermo internado em Unidades de Terapia Intensiva Neonatais, também desempenhando importante papel na formação de profissionais da área da saúde das universidades de Fortaleza e dos cursos técnicos de Enfermagem.

Os dados foram coletados entre os meses de agosto e outubro de 2007 por meio da aplicação de um formulário com questões referentes à identificação dos entrevistados e à dor no neonato. Participaram do estudo 180 profissionais de Enfermagem, todas do sexo feminino, sendo 45 enfermeiras, 74 técnicas e 61 auxiliares de enfermagem. Estas atenderam ao critério de inclusão da pesquisa: ter, no mínimo, um ano de atuação na área neonatal, o que garantiu pleno envolvimento com as rotinas de cuidado ao neonato criticamente doente.

Os dados coletados foram registrados no instrumento de coleta de dados, digitados em planilhas do programa Statistical Package for the Social Sciences - SPSS for Windows, versão 17.0, agrupados, codificados e processados. Foram utilizados, essencialmente, cálculos de somatório, médias aritméticas e percentuais.
Na análise dos dados, foi possível identificar relações entre as informações coletadas e remeter discussão de fundamentação teórica com base na revisão de literatura.

Esta pesquisa respeitou a condição humana e se propôs a cumprir os requisitos de autonomia, não maleficência, justiça e equidade, dentre as outras exigências explícitas na resolução 196/ 96 do Conselho Nacional de Saúde/Ministério da Saúde. Foi aprovada pelo Comitê de Ética em Pesquisa da Universidade Estadual do Ceará em 20 de agosto de 2007, sob o Processo n $07175881-0$, e pelos Comitês de Ética dos hospitais que constituíram os locais do estudo, sendo apreciados por seus membros e pelos responsáveis pelas coordenações das Unidades de Terapia Intensiva Neonatais, os quais concordaram com a realização da pesquisa.

\section{RESULTADOS}

No que diz respeito ao manejo da dor do neonato pela equipe de enfermagem, temática central deste estudo, as profissionais participantes foram questionadas se executavam medidas para minimizar/reduzir esta dor diante de procedimentos ou condições dolorosas diversas. Das entrevistadas, apenas duas (2) afirmaram não fazer uso de medidas para aliviar a dor do neonato.

As medidas citadas pelas entrevistadas, com as respectivas frequências absoluta e relativa, encontram-se listadas na Tabela 1.

Tabela 1. Medidas terapêuticas ou preventivas utilizadas pelos profissionais, e que, segundo eles, são eficientes no combate à dor no RN. Fortaleza-CE, 2007.

\begin{tabular}{lcc}
\hline \multicolumn{1}{c}{ Medidas } & N & $\%$ \\
\hline 1. Chupeta de gaze com glicose & 78 & 43,8 \\
2. Acalento & 42 & 23,6 \\
3. Pacotinho & 35 & 19,6 \\
4. Sucção não nutritiva & 9 & 5,0 \\
5. Chupeta de gaze com leite materno & 6 & 3,4 \\
6. Analgésico & 6 & 3,4 \\
7. Outras & 2 & 2,2 \\
8. Nenhuma & 2 & 2,2 \\
\hline Total & 180 & 100 \\
\hline
\end{tabular}

A comprovação de uma associação estatisticamente significante entre as variáveis "medidas para o alívio da dor" e "categoria profissional" $(p<0,012)$ é demonstrada na Tabela 2.

Tabela 2. Relação entre a implementação da medida para o alívio da dor do neonato e a respectiva categoria profissional. Fortaleza-CE, 2007.

\begin{tabular}{lccc}
\hline $\begin{array}{l}\text { Medida para } \\
\text { o al'vio da dor }\end{array}$ & $\begin{array}{c}\text { Enfermeiro } \\
n(\%)\end{array}$ & $\begin{array}{c}\text { Técnico de } \\
\text { Enfermagem } \\
n(\%)\end{array}$ & $\begin{array}{c}\text { Auxiliar de } \\
\text { Enfermagem } \\
n(\%)\end{array}$ \\
\hline $\begin{array}{l}\text { 1. Chupeta de gaze com glicose } \\
\text { 2. Acalento }\end{array}$ & $25(56,8)$ & $33(45,2)$ & $20(33,9)$ \\
3. Pacotinho & $8(18,2)$ & $17(23,3)$ & $17(28,8)$ \\
4. Sucção não nutritiva & $5(11,4)$ & $16(21,9)$ & $14(23,7)$ \\
5. Chupeta de gaze com leite materno & $6(13,6)$ & $1(1,4)$ & $2(3,4)$ \\
6. Analgésico & $0(0)$ & $4(5,5)$ & $2(3,4)$ \\
\hline
\end{tabular}




\section{Medidas para alívio da dor em Neonatos}

As justificativas atribuídas pelos profissionais para a implementação das medidas para o alívio da dor no neonato durante sua internação em uma UTIN são apresentadas na Tabela 3.

Tabela 3. Finalidades da utilização de medidas para o alívio da dor nos neonatos na opinião das entrevistadas. Fortaleza-CE, 2007.

\begin{tabular}{lcc}
\hline \multicolumn{1}{c}{ Justificativas } & N & $\%$ \\
\hline 1. Para minimizar o sofrimento & 151 & 84,8 \\
2. Porque a dor causa repercussões negativas à saúde do Rn & 8 & 4,5 \\
3. Para cumprir ordem da chefia (rotina do serviço) & 5 & 2,8 \\
4. Para oferecer um cuidado de Enfermagem de qualidade & 5 & 2,8 \\
5. Para facilitar o procedimento & 1 & 0,6 \\
6. Porque é um direito da criança ser tratada sem sentir dor & 1 & 0,6 \\
7. Outras razões & 7 & 3,9 \\
8. Nenhuma & 2 & 2,2 \\
\hline Total & 180 & 100 \\
\hline
\end{tabular}

\section{DISCUSSÕES}

Como pode ser visto na Tabela 1, a solução de glicose embebida em gaze foi citada pela maior parte das entrevistadas $(43,8 \%)$, que acreditam ser esta a principal medida a ser utilizada pela Enfermagem para acalmar o neonato e diminuir sua dor durante os procedimentos sabidamente dolorosos. Tal resultado indica que os profissionais demonstram conhecer 0 efeito benéfico da glicose para o neonato, que consiste na estimulação de opioides endógenos, ${ }^{1}$ acalmando-o antes dos referidos procedimentos, reduzindo sua reação à dor.

A administração de soluções adocicadas diretamente sobre a língua do neonato, anteriormente à realização de procedimentos dolorosos, tem sido importante para o neonato pré-termo ou neonatos gravemente enfermos, que sofrem múltiplos procedimentos invasivos e danos teciduais diariamente. ${ }^{8}$

Dentre as soluções mais estudadas, a sacarose e a glicose têm merecido destaque por apresentarem melhor efeito analgésico. Tal resultado pode ser observado pela diminuição na duração do choro, atenuação da mímica facial de dor, minimização da elevação da frequência cardíaca, além da diminuição dos escores de dor na aplicação da escala de Perfil de Dor do Prematuro (PDP) em neonatos a termo e prematuros. ${ }^{1,9}$

Embora evidenciados os efeitos benéficos descritos com relação à administração de soluções adocicadas, observa-se uma lacuna no que se refere à sua utilização em doses repetidas e por períodos prolongados, e para prematuros extremos ou neonatos gravemente enfermos. Isso pôde ser comprovado nesta pesquisa, em que as entrevistadas, apesar de conhecerem tais benefícios, não demonstravam certeza quanto à dose segura de glicose para ser oferecida ao neonato, antes de ele ser submetido ao procedimento doloroso. No entanto, sabe-se que o correto seria administrar $1 \mathrm{ml}$ de solução glicosada a 25\% por via oral 1 a 2 minutos antes dos procedimentos dolorosos. Em neonatos pré-termo, o volume mais adequado seria de 0,3 a $0,4 \mathrm{ml} .{ }^{10}$

Nesta pesquisa, também foi citado o uso do leite materno em chupeta de gaze para aliviar a dor no neonato, antes da sua submissão aos procedimentos dolorosos, porém por apenas 3,4\% das entrevistadas. Na verdade, a amamentação é um método analgésico aos neonatos saudáveis, conforme evidenciado por Gray et al. (2000) ${ }^{11} \mathrm{em}$ estudo prospectivo, randomizado e controlado, em que um grupo foi submetido a procedimento doloroso, como punção de calcanhar e coleta de sangue enquanto amamentava, e outro submetido ao mesmo procedimento, porém não amamentando durante 0 procedimento.

Os autores observaram ainda que o choro e as caretas reduziram em $91 \%$ e $84 \%$, respectivamente, durante o procedimento no grupo que estava amamentando em relação ao grupo-controle (11 de 15 RNs não choraram enquanto amamentavam ou não fizeram caretas na coleta de sangue). A frequência cardíaca reduziu também substancialmente. Os autores concluíram que a amamentação é, portanto, um potente analgésico durante a coleta de sangue nos neonatos.

Outra medida não farmacológica importante no tratamento e na prevenção da dor do RN, citada por 23,6\% da amostra, foi o Acalento, que consiste em transmitir carinho e proteção ao neonato por meio do toque, colocando-o no colo e, 
quando possível, conversando com ele. Em concordância com Lemos; Miguel (2008), ${ }_{12}^{12}$ acalentar ou oferecer a chupeta com solução glicosada diminuem a agitação e 0 estresse em momentos críticos como no ato de uma punção venosa no neonato, demonstrando que 0 ambiente e o propósito da equipe de enfermagem pode se associar ao uso de analgesia, minimizando e aliviando a dor destes pacientes.

As entrevistadas também relataram conhecer os benefícios do método mãe-canguru, que consiste em colocar o neonato despido, em contato com a pele da sua mãe, em posição ventral, amarrado sobre o seu tórax. Esteé um poderoso método de alívio da dor, conforme estudo realizado por Ahuja et al. $(2000)^{13}$. De acordo com a pesquisa diminuíram a expressão ou careta de dor em $65 \%$ e o choro em $82 \%$ durante a coleta do teste do pezinho, quando o neonato estava na posição canguru até 15 minutos antes do procedimento.

Em nosso estudo, as entrevistadas fizeram uso do termo "pacotinho" para especificar o aconchego, ou seja, colocar 0 neonato em posição confor tável e embrulhado, para que se sinta protegido durante o procedimento. Em estudo de Scochi et al. (2006) $)^{7}$, encontramos outra denominação para essa medida não farmacológica: "ninhozinho".

Outro estudo, em que foram avaliados 30 neonatos submetidos a uma punção capilar, 15 ficaram em contato pelepele antes, durante o procedimento e 3 minutos após, e o outro grupo controle de 15 foi submetido a coleta padrão no berço; 05 neonatos do grupo-caso (pele-pele) choraram menos durante a coleta e tiveram menos expressão facial de dor durante a punção e após o procedimento. Este achado parece ser explicado pela liberação de opioides endógenos levando a uma ação analgésica. ${ }^{5}$

Dessa forma, o método mãe-canguru demonstra ter uma grande vantagem para evitar a dor neonatal. Nesse contexto, quando possível, ressaltamos a importância da presença da mãe nos cuidados ao neonato, pois é ela quem permanece o maior período no hospital, como mulher cuidadora, responsável pela saúde e educação dos filhos. Uma variável adicional de impacto é que, quanto mais instrução a mãe tiver, mais facilidade ela terá de aprender 0 cuidado e de executá-10.14

No que diz respeito ao toque, em neonatos fisiologicamente instáveis, o toque de afago (alisamento repetitivo em uma única direção) pode provocar dessaturação de oxigênio e estresse comportamental. ${ }^{15}$ Já o toque contensivo, caracterizado pela sobreposição das mãos no corpo do neonato, tem um efeito positivo na melhoria do estresse sofrido na internação em uma UTIN.

Outra medida citada por 42 entrevistadas $(23,6 \%)$ foi 0 acalento. Elas relataram que conversar com o neonato antes dos procedimentos é uma maneira de minimizar a sensação de dor e de tranquilizá-lo para a execução das técnicas.

Estudo recente realizado com 116 profissionais que atuam em UTINs ${ }^{15}$ evidenciou que $19 \%$ destes referiram conversar com o bebê como forma de tranquilizar ou avisar sobre o procedimento realizado.

Autores ${ }^{16}$ relatam que falar com o bebê permite sua inserção no mundo da linguagem, principalmente quando 0 som é originário da voz materna, o que pode representar um amparo psíquico ao bebê. Mesmo não compreendendo 0 significado das palavras, o recém-nascido é capaz de captar a melodia das mesmas, aprendendo a forma pelo qual são expressos os sentimentos.

Neste estudo, a sucção não nutritiva foi citada por apenas $5 \%$ das profissionais entrevistadas. Em recente estudo realizado com 116 profissionais que atuam exclusivamente na UTIN, entre médicos e de enfermagem, foi encontrado que $34 \%$ dos profissionais indicavam a sucção não nutritiva como paliativo em procedimentos dolorosos. ${ }^{15}$

Em estudo de Lamego et al. (2005) ${ }^{17}$, embora houvesse restrições institucionais estabelecidas quanto ao uso de chupetas, parte da equipe mostrou-se favorável ao seu uso nos bebês submetidos a cirurgias, pois eles ficam privados, nos períodos pré e pós-operatórios, do efeito tranquilizador proporcionado pela sucção não nutritiva em situações de dor e estresse. Não eram utilizadas chupetas convencionais, mas sim bicos improvisados com luvas esterilizadas, guarnecidas internamente com algodão.

Por ser um reflexo natural do neonato, a sucção não nutritiva é uma das medidas comportamentais mais frequentemente utilizadas pela equipe de enfermagem atuante em UTINs, pois cer tamente promove calma e conforto aos neonatos. ${ }^{1}$ Sua ação está relacionada à diminuição do tempo de choro resultante de lancetagem de calcâneo, bem como à elevação da frequência cardíaca menos significativa, em resposta ao procedimento.

Indicada em procedimentos como punção venosa e lancetagem de calcâneo, "a sucção não nutritiva inibe a hiperatividade e modula o desconfor to do neonato, além de diminuir a dor em RN a termo e prematuros submetidos a procedimentos dolorosos agudos".

Os efeitos da sucção não nutritiva associam-se ao aumento na oxigenação, à melhora nas funções respiratória e gastrintestinal e à diminuição da frequência cardíaca e do gasto energético, promovendo descanso e analgesia.

Ao analisarmos a Tabela 2, constatamos que a maioria das enfermeiras $(56,8 \%)$ e a maior parte das técnicas $(45,2 \%)$ e auxiliares de Enfermagem $(33,9 \%)$ utilizam a chupeta de gaze embebida em glicose como a principal medida para a redução da dor diante dos procedimentos dolorosos. Isto demonstra a eficácia do uso desta solução, que deve ser considerada durante 0 procedimento doloroso para que o neonato fique mais calmo e preparado para tal.

Quanto às justificativas atribuídas à implementação de medidas para 0 alívio da dor, apresentadas na Tabela 3, a maioria das entrevistadas $(84,8 \%$ ) relatou que executa tais 


\section{Medida para alívio da dor em Neonatos}

medidas para minimizar/aliviar o sofrimento do bebê, demonstrando sentimentos de pena. Nesse contexto, observase a vinculação profissional-bebê, que é considerado elemento fundamental para o bebê reorganizar-se emocional e fisiologicamente após a realização de intervenções clínica. ${ }^{17}$ Essa vinculação é facilmente observada nas situações em que uma forte ligação é estabelecida entre 0 profissional e 0 neonato, principalmente nos casos mais graves e na ausência materna.

Justificativas adicionais foram citadas em frequência bem menor, destacando-se a aplicação de medidas para minimizar os efeitos negativos produzidos pela dor na saúde do neonato a longo prazo, citadas por algumas poucas entrevistadas (4,5\%). Ressaltamos que tal justificativa foi citada apenas por enfermeiras. Autores ${ }^{18}$ afirmam que 0 fato de enfermeiros almejarem uma atenção mais humanizada durante os procedimentos dolorosos leva-os a intervir de forma vigorosa, de modo a atenuar esse sofrimento, minimizando as consequências da dor.

Uma justificativa que também nos chama atenção é a de cumprir ordem da chefia do serviço, apontada por cinco entrevistadas $(2,8 \%)$. Com esta resposta, podemos pensar na possibilidade de estas profissionais desenvolverem suas atividades assistenciais como cumprimento de protocolos existentes na unidade, caracterizando uma possível falta de vínculo com o serviço. Isso demonstra que, mesmo inseridos no contexto de humanização da assistência, e principalmente em se tratando de unidades que valorizam este aspecto, existem profissionais que executam um cuidado instrumentalizado, fragmentado e sem envolvimento, fato que precisa ser revisto nas unidades estudadas.

\section{CONCLUSÕES}

Demonstrando uma assistência diferenciada e que visa à qualidade dos cuidados ao neonato, as entrevistadas citaram medidas não farmacológicas consideradas importantes na prevenção da dor anteriormente aos procedimentos dolorosos, destacando-se: chupeta de gaze embebida em glicose, acalento, pacotinho, sucção não nutritiva e chupeta de gaze embebida em leite materno. Tais medidas são frequentemente divulgadas na literatura como eficientes, devendo seu uso ser continuamente estimulado nas unidades neonatais juntos aos profissionais de enfermagem.

No contexto do estudo em tela, as entrevistadas demonstraram motivação para a prestação de cuidados aos neonatos, ao relatarem a utilização dessas medidas eficazes para a prevenção da dor mediante procedimentos invasivos e sabidamente dolorosos. Portanto, a maioria apresentou conhecimento sobre a eficácia de medidas não farmacológicas para tratamento e prevenção da dor, o que indica um resultado positivo e que deve ser estimulado na rotina de cuidados neonatais.
Entretanto, apesar do incentivo às políticas de humanização das práticas de saúde nas unidades neonatais, percebemos que algumas profissionais de enfermagem ainda exercem um cuidado centrado nas técnicas e nos protocolos do serviço.

Concluímos que há necessidade de reflexões sobre 0 conhecimento teórico-prático dos cuidados com o neonato em situações dolorosas, incluindo identificação das fácies e repercussões fisiológicas da dor, pois foi verificada certa inconsistência entre os saberes e as práticas. Destacamos 0 fato de as entrevistadas não demonstrarem certeza quanto à dose segura de glicose a ser oferecida ao neonato como medida analgésica de excelência utilizada pela enfermagem no alívio da dor.

Ressaltamos, ainda, a importância e a necessidade de estudos subsequentes sobre a temática, assim como 0 desenvolvimento de protocolos de cuidados ou intervenções de enfermagem junto ao neonato em situações de enfrentamento da dor.

\section{REFERÊNCIAS}

1. Bueno M. Dor no período neonatal. In: Chaves LD, Leão ER. Dor: 50 sinal vital: reflexões e intervenções de enfermagem. Curitiba: Maio; 2004. p.194-205.

2. Chermont AG, Guinsburg R, Balda RCX, Kopelman BI. 0 que os pediatras conhecem sobre avaliação e tratamento da dor no recém-nascido? J Pediatr. 2003; 79(3):265-72.

3. American Academy of Pediatrics. American Pain Society. The assessment and management of acute pain in infants, children and adolescents. Pediatrics. 2001; 108: 793-97.

4. Okada M, Teixeira MJ. Desenvolvimento do sistema nociceptivo e supressor da dor. In: Teixeira MJ, Filho JLB, Marquez JO, Yeng LT. Dor: contexto interdisciplinar. Curitiba: Maio; 2003. p.89-117.

5. Margotto PR, Rodrigues DN. Dor neonatal: analgesia/sedação. In: Margotto PR. Assistência ao recém-nascido de risco. $2^{\text {aed. }}$. [sl]: Ed Autor; 2004.

6. Pimenta CAM. Dor: Manual Clínico de Enfermagem. São Paulo[sn]; 2000.

7. Scochi CGS, Carletti M, Nunes R, Furtado MCC, Leite AM. A dor na unidade neonatal sob a perspectiva dos profissionais de enfermagem de um hospital de Ribeirão Preto-SP. Rev Bras Enferm. 2006; 59(2):18894.

8. Johnston CC, Filion F, Snider L, Majnemer A, Limperopoulos C, Walker CD. et al. Routine sucrose analgesia during the first week of life in neonates younger than 31 weeks' postconceptional age. Pediatrics. 2002; 110(3): 523-28. 
9. Guinsburg R, Balda RCX. Dor em neonatologia. In: Teixeira MJ. Dor: contexto interdisciplinar. Curitiba: Maio; 2004. p.548-54.

10. Medeiros MD, Madeira LM. Prevenção e tratamento da dor do recémnascido em terapia intensiva neonatal. REME: Rev Min Enferm. 2006; 10(2):118-24.

11. Gray L, Watt L, et al. Skin-to-skin contact is analgesic in healthy newborns. Pediatrics. 2000 jan.; 105(1):14.

12. Lemos S, Miguel EA. Caracterização do manejo da dor, realizado pela equipe de enfermagem, na unidade de terapia intensiva pediátrica. Cienc Cuid Saude. 2008; 7(1):82-7.

13. Ahuja VK, Daga SR, Gosavi DV. et al. Non-sucrose sweetner for pain relief in sick newborns. J Pediatr. 2000;67(7):487-89.

14. Sousa JC, Silva LMS, Guimarães TA. Preparo para a alta hospitalar de recém-nascido em unidade de tratamento intensivo neonatal: uma visão da familia. Pediatria. 2008; 30(4):217-27.

15. Hennig MAS, Gomes MASM, Glanini NOM. Conhecimentos e práticas dos profissionais de saúde sobre a atenção humanizada ao recém-nascido de baixo peso- Método Canguru. Rev Bras Saude Matern Infant. 2006 out/dez; 6(4): 427-35.

16. Braga NA, Morsch DS. Os primeiros dias na UTI. In: Moreira MEL, Braga NA, Morsch DS, organizadores. Quando a vida começa diferente: 0 bebê e sua familia na UTI neonatal. Rio de Janeiro: Fiocruz; 2003. p.51-68.

17. Lamego DTC, Deslandes SF, Moreira MEL. Desafios para a humanização do cuidado em uma unidade de terapia intensiva neonatal cirúrgica. Cienc Saude Colet. 2005; 10(3):669-75.

18. Silva TM, Chaves EMC, Cardoso MVLM. Dor sofrida pelo recémnascido durante a punção arterial. Esc Anna Nery. 2009 out/dez; 13(4): 726-32. 